\title{
The Importance of Physical Diagnosis in Internal Medicine
}

With the recent developments in science and technology, medicine has witnessed significant progress. The result is a dramatic advancement in specialization in internal medicine and a growing trend toward further compartmentalization so that the question arises as to what internal medicine should be like.

Here, I would like to address may view on this subject.

\section{THE BASIS OF INTERNAL MEDICINE}

Internal medicine is the mainstream of medicine. It is medicine from a broad field of view. Thus, it is not possible to achieve the inherent tasks of internal medicine exclusively on the basis of a very specialized approach with knowledge limited to a very particular area of internal medicine and without being familiar with other fields. I think that internal medicine is a practical medical discipline based on a broad knowledge extending to medical science as a whole and trying to determine the interior causes of disease, establishing the correct diagnosis, and undertaking the appropriate therapy. It is not limited to one particular field of clinical medicine and is essentially the basis of clinical medicine uniting many special disciplines.

Indeed, Professor Hideo Ueda, Tokyo University School of Medicine, defined the essence of internal medicine as a basic discipline of clinical medicine, the purpose of which is to elucidate the condition or status of the disease and to apply the appropriate therapy so as to treat the patient's disease quickly.

\section{THE IMPORTANCE OF PHYSICAL DIAGNOSIS}

Mordern diagnostic techniques with the latest sophisticated diagnostic apparatus and methods have reached outstanding accuracy in morphological or functional diagnostic terms. However, diagnosis done with this kind of apparatus is restricted to the particular organ or the particular organs under examination, while the patient's general pathological development, the presence of complications or the existence of pathological changes in the organ or organs other than those examined often tend to be left out of consideration. To reduce this oversight, it is essential to perform physical diagnosis and to review the patient's condition systematically through general observation.

I have had the great pleasure of seeing the completion of several revised editions of my original book, Physical Diagnosis, which was published in 1966. In the 1976 revised edition of the book, I introduced the concept of diagnostic records taken within the framework of a problem-oriented system. In the revised edition of 1984, I re-emphasized the importance of a diagnostic approach that observes the body as a whole, including a systematic review of the patient's overall, general symptoms. I have believed that the correct diagnosis can only be arrived at through detailed diagnostic observation with the disease being established by a process of careful elimination from an overall assessment of the data obtained from the patient's medical history, his presenting symptoms, and clinical examination on the basis of a correctly applied physical diagnosis.

Physical diagnosis is essential to a proper appreciation of the patient's psychological and somatic pain, his suffering in mind and body, and to a correct understanding of his overall state of illness. Diagnosis based on the findings obtained from taking the patient's medical history and observing his presenting symptoms ensures that the patient is not subjected to any unnecessary examinations and that his is spared any untoward psychological, physical, and financial burden.

\section{Jugoro TAKEUCHI}

President of the Japanese Society of Internal Medicine (1985) and Professor of Internal Medicine, The Second Department of Internal Medicine, School of Medicine, Tokyo Medical and Dental University, Tokyo 